\title{
E Research Square \\ Identification of Key Genes and Pathways in
Hypertrophic Cardiomyopathy via Weighted Gene Co-Expression Network Analysis
}

\section{Yang Hua}

Jiangsu Province Hospital and Nanjing Medical University First Affiliated Hospital Jin-Yu Sun

Jiangsu Province Hospital and Nanjing Medical University First Affiliated Hospital

Ziling Xin

Jiangsu Province Hospital and Nanjing Medical University First Affiliated Hospital

Wei Sun

Jiangsu Province Hospital and Nanjing Medical University First Affiliated Hospital

Yanhui Sheng

Jiangsu Province Hospital and Nanjing Medical University First Affiliated Hospital

Xiang-Qing Kong ( $\triangle$ kongxqnjmu@163.com )

Jiangsu Province Hospital and Nanjing Medical University First Affiliated Hospital https://orcid.org/0000-0003-0163-9650

\section{Research}

Keywords: hypertrophic cardiomyopathy, weighted gene co-expression network analysis, differentially expressed genes, integrated bioinformatics analysis

Posted Date: August 2nd, 2021

DOI: https://doi.org/10.21203/rs.3.rs-723383/v1

License: (1) This work is licensed under a Creative Commons Attribution 4.0 International License. Read Full License 


\section{Abstract}

\section{Background}

Hypertrophic cardiomyopathy $(\mathrm{HCM})$ is a prevalent cardiovascular disease characterized as asymmetric hypertrophy of ventricular muscles. Cardiac morphological abnormality may result in slight or severe cardiopulmonary symptoms, arrhythmia, heart failure, and even sudden death. Previous studies have shown that HCM was an inherited disease where sixty percent carry mutations in genes encoding sarcomere proteins. However, considering heterogeneous phenotype or prognosis, the underlying mechanisms remain unclear.

\section{Methods}

The gene expression profiles of GSE36961 and GSE160997 were analyzed by 'limma' and 'weighted gene co-expression network analysis (WGCNA)' package in R to identify differentially expressed genes (DEGs) and key modules, respectively. Then, enrichment analysis was performed based on Gene ontology (GO) and Kyoto Encyclopedia of Genes and Genomes (KEGG) pathway. Protein-protein interaction network was constructed based on the overlapped genes of DEGs and key modules, and we identified the top 4 hub genes using 'cytohubba' according to inner connectivity.

\section{Results}

We identified the red and brown modules as the key modules. Enrichment analysis showed that cellular divalent inorganic cation homeostasis, collagen-containing extracellular matrix, and actin binding were significantly enriched. VSIG4, CD163, FCER1G, and LAPTM5 were identified as hub genes.

\section{Conclusions}

This study suggested that VSIG4, CD163, FCER1G, and LAPTM5 might be hub genes associated with the progression of HCM. Further studies are required to elucidate the underlying mechanisms and provide potential therapeutic targets.

\section{Introduction}

Hypertrophic cardiomyopathy $(\mathrm{HCM})$ is a common inherited cardiovascular disease with a prevalence of at least one in $500(0.2 \%)$ in general[1]. Clinical feature is such abnormal cardiac morphology as unexplained asymmetric left-ventricular hypertrophy indicated by echocardiography or cardiovascular MRI, leading to arrhythmia, heart failure, and even sudden death in young people. However, the majority of affected individuals probably remain undiagnosed with normal life expectancy and few substantial symptoms[2]. Current treatments for HCM follows risk-stratification guidelines, including longitudinal follow-up in asymptomatic patients, pharmacologic treatment, ventricular septal surgery in patients with outflow obstruction, ICD implantation for prevention of sudden death in high-risk group, and heart transplantation in patients with end-stage heart failure[3]. 
With the rapid development of automated DNA sequencing technology, pathogenic mutations behind hypertrophic cardiomyopathy were largely researched over the past 20 years[4,5]. Genetic studies showed that HCM was associated with dominant mutations in genes encoding thick and thin contractile myofilament protein components of the sarcomere or the adjacent Z-disc[6-9]. However, the molecular mechanism underlying the pathogenesis of HCM is yet unclear. What's most ambiguous is unexplained pathological and phenotypic heterogeneity among individuals. For example, heterogeneity among racial and ethnic populations make it difficult to discriminate between pathogenic and nonpathogenic individuals[10,11]. Moreover, phenotypic heterogeneity in morphological features such as mitral-valve enlargement[12, 13], microvascular abnormalities[14], and segmental left-ventricular hypertrophy[15] results in various clinical charaeteristics, suggesting that HCM phenotype are not determined by a single gene but by the synergy in a complex network.

Gene co-expression network-based approaches have been widely used in analyzing microarray data, especially for identifying functional modules[16]. WGCNA (weighted gene co-expression network analysis) is one of the most useful gene co-expression network-based approaches and has been conducted to assess the association between gene sets and external sample characteristics by building a scale-free gene co-expression network[17]. Thus, we used the WGCNA algorithm to recognize networkcentric genes in the occurrence and development of HCM.

\section{Materials And Methods}

\subsection{Data collection}

The gene expression profiles of GSE36961 (https://www.ncbi.nlm.nih.gov/geo/query/acc.cgi? acc=GSE36961) and GSE160997 (https://www.ncbi.nlm.nih.gov/geo/query/acc.cgi?acc=GSE160997) were acquired from the Gene Expression Omnibus (GEO) database. Dataset GSE36961 was performed based on the platform GPL15389 (Illumina HumanHT-12 V3.0 expression beadchip), which includes 109 surgical myectomy tissue from patients with HCM and 36 normal samples. Series GSE160997 was performed by GPL11154 (Ilumina HiSeq 2000 (Homo sapiens), which contains 18 DNA whole-exome sequencing (WES) data and 23 RNA-seq transcriptome data. We only acquired 23 RNA-seq transcriptome data which includes 18 anterior septal tissues from HCM patients and 5 left ventricular wall tissues from healthy controls. Moreover, we also collected clinical information including age, gender, and type for further analysis.

\subsection{Data processing and DEG screening}

"LIMMA" package in R was employed to recognize differentially expressed genes (DEGs) between samples[18]. The probe IDs were converted into gene symbols according to the annotation file. For multiple probes mapping to a single gene, the average expression value of all its corresponding probes was used. To minimize the possible false discovery rate resulted from multiple testing, the adjusted $P$ value was applied. DEGs were screened with a threshold of adjusted $P$ value $<0.05$ and $\| \log _{2}$ fold-change 
$(F C) \mid \geq 0.5$. Furthermore, the DEGs were visualized as a volcano plot and heatmap using 'ggplot2' and 'pheatmap' package in $\mathrm{R}$.

\subsection{Weighted gene co-expression network analysis (WGCNA)}

WGCNA is an algorithm for constructing a co-expression network, which reveals the correlation patterns across genes and provides the biologically functional interpretations of network modules. We selected the top 5000 most variant genes in GSE36961 to construct a co-expression network using the 'WGCNA' package (version 1.60). After we evaluated the presence of obvious outliers by cluster analysis, the onestep network construction function was used to construct the co-expression network and identify key modules. Genes were hierarchically clustered and visualized in a dendrogram. The branches of the tree with the same color represented one specific module with highly correlated genes. Genes coloring gray were not belonged to any of the modules. Moreover, to identify the significance of each module, we summarized the module eigengene (ME) based on the first principal component of the module expression, and the Module-trait relationships were assessed according to the correlation between MEs and clinical traits. Then, we evaluate the correlation strength by module significance (MS), which is the average absolute gene significance (GS) of all genes within one module. Importantly, the GS value was determined by the $\log _{10}$-transformation of the $\mathrm{P}$ value in the linear regression between expression and clinical traits. In general, the modules with the highest MS values were considered as the key modules.

\subsection{Enrichment analysis of genes in key modules}

To understand the biological meaning of the genes in key modules, we used the 'clusterProfiler' package in R to perform Gene Ontology (GO) and Kyoto Encyclopedia of Genes and Genomes (KEGG) pathway enrichment analysis. The $\mathrm{GO}$ terms were consisted of 3 components: biological processes (BP), molecular functions (MF), and cellular components (CC). The biological functions of GO terms and KEGG charts were enriched with $p<0.05$. Aditionally, we used Metascape [19]to further divide genes into a subset of terms (with a similarity of $>0.3$ ) and visualize the association between terms and pathways, coloured by cluster ID. Furthermore, DisGeNET[20] and TRRUST [21]database were employed to respectively perform enrichment analyisis on clinical diseases and transcriptional regulatory networks level.

\subsection{PPI network construction and identification of hub genes}

In WGCNA, we defined the genes from key modules with $|\mathrm{MM}| \geq 0.8$ and $|\mathrm{GS}| \geq 0.2$ as key genes. We used the online Search Tool for the Retrieval of Interacting Genes (STRING database, Version 10.5, http://string-db.org/) to construct a protein-protein interaction (PPI) network[22]. After overlapping the DEGs and key genes from WGCNA, we input these genes into STRING database to collect interaction of target proteins with a medium confidence score of $>0.4$ and constructed a protein-protein interaction (PPI) network by Cytoscape software (v3.7.2)[23]. Further, we screened out the top 10 genes using the Cytoscape plug-in software 'cytoHubba' based on calculation of degree. After reviewing literature, hub genes were identified. 


\section{Results}

\subsection{Identification of differentially expressed genes}

In GSE36961, 899 genes were found to be differentially expressed between HCM samples and control samples. We identified 372 up-regulated and 527 down-regulated genes (dataset1). The volcanic diagram for all genes and the expression heat map of the top 25 DEGs are shown in Figure 1A-B. In GSE160997, 429 genes were up-regulated, and 4350 genes were down-regulated (Figure 1C-D) (dataset2).

\subsection{Weighted co-expression network construction and key modules identification}

With a scale-free network and topological overlaps, a hierarchical clustering tree was created based on the dynamic hybrid cut (Figure 2A-B). As previously described, the expression values of top 5000 most variant genes in GSM36961 were analyzed to identify the modules of highly correlated genes. The soft threshold power was set at 7 (scale-free $\mathrm{R}^{2}=0.98$, Figure $2 \mathrm{C}$ ) to guarantee a scale-free network. Using WGCNA package in $\mathrm{R}$, the genes with similar patterns of expression were grouped into modules via hierarchical average linkage clustering (Figure 2D). A total of 15 modules were identified, including black (191 genes), blue (916 genes), brown (776 genes), cyan (43 genes), green (248 genes), greenyellow (102 genes), grey (317 genes), magenta (143 genes), pink (181 genes), purple (116 genes), red (220 genes), salmon (50 genes), tan (54 genes), turquoise (1189 genes), and yellow (454 genes). The genes in grey were not included in any module, so no further analysis was conducted for these genes. The heat map depicting the TOM of genes is shown in Figure 3-A.

Moreover, the modules were analyzed with clinical traits, including disease status, age, and sex. The gene significance across modules was shown in Figure 3B-C. The red and brown modules were the two modules most significantly correlated with $\mathrm{HCM}$, and therefore, these two modules were identified as key modules accordingly. A total of 220 and 776 genes were included, respectively. In Figure 4A-B, we illustrated the correlation between module membership and GS in red (correlation coefficient $=0.78, P=$ 3e-26) and brown modules (correlation coefficient $=-0.91, P=5 e-56$ ), respectively.

\subsection{Enrichment analysis of key modules}

We performed functional enrichment analysis on the red and brown modules based on GO and KEGG databases. As shown in Figure 4C-D, enriched BPs were mainly involved in cellular divalent inorganic cation homeostasis, neutrophil mediated immunity, neutrophil degranulation, extracellular structure organization, and transition metal ion homeostasis. The cellular components (CCs) were mainly enriched in collagen-containing extracellular matrix and vesicle lumen. Enriched molecular functions (MFs) were mainly involved in actin binding. KEGG pathway analysis showed that the phagosome and Kaposi sarcoma-associated herpesvirus infection pathways were the most enriched pathways, followed by cellular senescence, chemokine signaling pathway, and complement and coagulation cascades. Metascape Analysis confirmed that the genes in key modules played a vital role in regulated exocytosis, and the network of enriched terms is shown Figure 4E. 
Moreover, as shown in Figure 4F, the enrichment analysis in DisGeNET showed that the genes in red and brown modules were significantly associated with IGA glomerulonephritis, bactria infections, and lupus nephritis. TRRUST database witnessed multiple transcription factors associated with the genes in key modules, including SPI1, SP1, RELA, NFKB1, HIF1A and ETS1.

\subsection{Identification of hub genes and PPI network}

With a threshold of $|\mathrm{MM}| \geq 0.8$ and $|\mathrm{GS}| \geq 0.2,15$ and 86 key genes were identified from the red and brown modules, respectively. After overlapping the DEGs (dataset1 \& dataset2) and key genes, a total of 44 genes were screened out (Figure 5A). Moreover, to explore the mechanisms of these hub genes in the development of HCM, a PPI network was created (Figure 5-B). CytoHubba revealed the top 10 hub genes according to calculation of degree connectivity (Figure 5-C). After reviewing literature, 4 hub genes were identified at last, including V-set and immunoglobulin domain-containing protein 4 (VSIG4), CD163, high affinity immunoglobulin epsilon receptor subunit gamma (FCER1G), and Lysosomal-associated transmembrane protein 5 (LAPTM5).

\section{Discussion}

Hypertrophic cardiomyopathy (HCM) is the most common genetic heart disease and associates with mutations in sarcomeric genes. Nearly half of HCM patients with positive genetic tests would carry mutations in genes encoding sarcomeric proteins, which were important in regulating cardiomyocyte contraction and cardiac function[24, 25]. However, variable penetrance and expressivity of HCM patients (even in the same family) implies that specific mechanism behind HCM is complicated and many factors such as epigenetic modifiers or environmental influences may affect the phenotype, as witnessed by previous researches.[26, 27]. At present, no treatments have been found to completely stop the progression and cure the disease. Therefore, it is of great significance to understand etiological explanations for HCM and explore new effective therapeutic strategies.

In this study, we identified co-expression genes and possible biological pathways of HCM, aimed to detect hub genes and facilitate new therapy in the future. We acquired 2 datasets from GEO database and identified 899 and 4,579 DEGs with R package, respectively. To further research the association between genes and external clinical features, we selected top 5000 variable genes of GSE36961 to carry out a WGCNA study. 15 different modules were identified and genes clusterd into the red and brown module were mostly associated with HCM. Functional enrichment analysis indentified cellular divalent inorganic cation homeostasis, neutrophil mediated immunity, and neutrophil degranulation to be highly engaged in the status of HCM. With a threshold of $|\mathrm{MM}| \geq 0.8$ and $|\mathrm{GS}| \geq 0.2,44$ and 86 key genes were chosen from the red and brown modules, respectively. We later overlapped the DEGs (dataset1 \& dataset2) and key genes. After choosing genes with high connectivity using the Cytoscape plug-in software 'cytoHubba' based on calculation of degree and researching entensive literature, 4 genes including VSIG4, CD163, FCER1G, and LAPTM5 were finally identified as hub genes of HCM. 
The V-set and immunoglobulin domaincontaining protein 4 (VSIG4) is a member of complement receptor of the immunoglobulin superfamily (CRIg), mainly expressed on macrophages[28]. Association between VSIG4 and macrophage was largely researched. Jialin Li et al[29] demonstrated that VSIG4 could inhibit macrophage activation through PI3K/Akt-STAT3 pathway. Xiaoyong Huang et al[30] also verified that VSIG4 is specifically expressed in non-activated macrophages and mediates inhibitory signals to suppress the transcription of Nlrp3 (Nod-like receptor protein 3), an important inflammasome engaged in pathogenesis of various inflammatory syndromes including cardiovascular disease. Cardiac remodeling is a major pathological process in the progression of HCM and the role of macrophages in cardiac remodeling has been largely studied. Sarah Kitz et al compared 18 feline HCM cases to 18 cats without $\mathrm{HCM}$ and suggested that HCM is the consequence of cardiac remodeling processes in which macrophages were involved by maintaining an inflammatory and profibrotic environment[31]. Specifically, CCR2+ macrophages are able to produce and secrete substantial proinflammatory cytokines, including NLPR3 and IL-1 $\beta$, to facilitate adverse cardiac remodeling such as hypertrophy, fibrosis, and LV dilation[32]. However, the role of macrophages in cardiac remodeling is not absolute. Take the role of monocytes and macrophages in fibrotic response as an example. 'Pro-fibrotic' macrophage may alter the extracellular matrix by producing cytokines (TNF-a, IL-1 $\beta$, IL-10), chemokines (MCP-1) and growth factors including TGF $\beta$ and PDGF (platelet-derived growth factor) to activate transformation of cardiac fibroblasts into myofibroblasts and therefore promote the fibrotic response[33, 34]. On the other hand, some macrophages, we call them 'anti-fibrotic' macrophages, could restrain fibrosis by removing apoptotic myofibroblasts and suppressing fibroblast activation[35, 36]. In our study, we observed a decreased expression of VSIG4 in HCM tissue compared to normal heart.

$\mathrm{CD} 163$, also known as the hemoglobin $(\mathrm{Hb})$ scavenger receptor, is a 130-kDa membrane protein with a short cyto-plasmic tail, a single transmembrane segment, and a large ectodomain[37]. Being a macrophage-specific protein, CD163 is restricted to the monocytic-macrophage linage such as red pulp macrophages, bone marrow macrophages, and liver macro-phages (Kupffer cells). [38]. CD163 has been verified to indirectly contribute to atheroprotective and anti-inflammatory response through scavenging of the oxidative $\mathrm{Hb}$, stimulation of the heme-oxygenase- 1 and production of anti-inflammatory heme metabolites[39]. However, apart from its overall anti-inflammatory function, CD163 could also produce inflammatory cytokines, for example TNF-a and IL-4, in inflammation response. The upregulated expression of this receptor reflects the switch of macrophage to activated phenotypes in inflammation and thus, a high CD163 expression in macrophages reflects the activated response of tissues to inflammation[40]. Enhanced expression of CD163+ or soluble CD163+ macrophages were detected in human inflammatory diseases including chronic cardiovascular disease like atherosclerosis[41], atrial fibrillation[42, 43], and chronic heart failure[44, 45]. Therefore, novel evidence indicated CD163 a potential inflammation biomarker and a therapeutic target in a large spectrum of acute and chronic inflammatory disorders[46, 47]. Accumulating evidence has suggested the existence of low-grade systemic and local inflammation in HCM. Chronic inflammatory cell infiltration was observed in the myocardium of patients with $\operatorname{HCM}[48,49]$. Lu Fang et al [50] observed an increasing expression of tumor necrosis factor (TNF)-a, interleukin (IL)- 6 and serum amyloid P (SAP) in HCM patients compared to controls and that systemic 
inflammation is related to the severity of HCM patients, especially regional and diffuse fibrosis. They also suggested that mechanic stress could upregulate NF-KB nuclear positivity in cardiac cells, fueling the inflammatory process and transdifferentiation of fibroblast to active myofibroblast, the main contributor of cardiac collagen. To conclude, modifying inflammation may reduce myocardial fibrosis in HCM patients.

FCER1G is localized on chromosome 1q23, which encodes the $y$ subunit of the fragment crystallizable $(F c)$ region ( $F c R$ ) of immunoglobulin $E(\mathrm{IgE})$. In normal immune fuction of human body, Fc binds to $F c R$ of immune cells to recognize and eliminate extraneous antigens via the antigen-antibody binding reaction. However, such combination may generate adverse effects as it may trigger destructive inflammation, immune cell activation, phagocytosis, oxidative burst, and cytokine release under pathological circumstance[51]. Sandra B. Haudek reported that serum amyloid P (SAP) could prevent cardiomyopathy in the heart by binding to Fc receptors (FcRs) expressed on monocytes and inhibiting monocyte-to-fibroblast transition. Mice lacking the FcRy, a common membrane-signaling component of activating FcRs developed fibrosis and cardiac dysfunction when subjected to a murine fibrotic cardiomyopathy model[52]. It was reported that FcRY-Chain deficiency reduces the development of dietinduced obesity[53] and possible mechanism may be antibody-mediated immune responses involved in the development of insulin resistance. The normal physiological activity of heart requires a large amount of energy and there is increasing evidence of the link between metabolism and cardiac hypertrophy, especially oxidation of fatty acid and glucose[54]. Liling Zheng et al [55]found that the ability of energe utilization was impaired in hypertrophic hearts manifested as reduced fatty acid oxidation rates and myocardial insulin resistance. Mitochondrial function and cardiac systolic function in hypertrophic hearts were also disrupted. Owing to possible correlation between metabolic dysfuction and cardiac hypertrophy, FCER1G may be a target for further research.

Lysosomal-associated protein multispanning transmembrane 5 (LAPTM5), a membrane protein highly expressed in lymphoid lineage cells, plays a fundamental role in mediating vesicle trafficking [56]. LAPTM5 was associated with inhibition of T cell receptor (TCR) [57], B cell receptor (BCR)[58], and pre-B cell receptor (pre-BCR)[59] expression on the cell surface via promotion of lysosomal degradation of these proteins, indicating its negative regulator in immunization. Moreover, association between LAPTM5 and inflammation in macrophages was reported by Glowacka WK[60]. LAPTM5 was a positive regulator of multiple inflammatory cascades in the activation of macrophages through NF-KB and MAPK pathway and downstream proinflammatory cytokine release (IL6, TNF, and IL12). A large number of studies have been carried on LAPTM5 and human cancer[61, 62], but little on LAPTM5 and cardiovascular disease. In the future, exploration of LAPTM5 may be targeted as a new direction for research on HCM.

\section{Conclusions}

This study suggested that VSIG4, CD163, FCER1G, and LAPTM5 might be hub genes associated with the progression of HCM. Further studies are required to elucidate the underlying mechanisms and provide novel therapeutic targets. 


\section{Abbreviations}

HCM: Hypertrophic cardiomyopathy, WGCNA:Weighted gene co-expression network analysis, DEGs: Differentially expressed genes, GO and KEGG pathway: Gene ontology and Kyoto Encyclopedia of Genes and Genomes pathway, GEO: Gene Expression Omnibus, WES: Whole-exome sequencing, ME: Module eigengene, MS: Module significance, GS: Gene significance, BP: Biological process, MF: Molecular functions (MF), CC: Cellular components, PPI: Protein-protein interaction, VSIG4: V-set and immunoglobulin domaincontaining protein 4, FCER1G: High affinity immunoglobulin epsilon receptor subunit gamma, LAPTM5: Lysosomal-associated transmembrane protein 5

\section{Declarations}

\section{Ethics approval and consent to participate}

Not applicable

\section{Consent for publication}

Not applicable

\section{Availability of data and material}

GEO database is a public accessible database containging human gene expression data. The gene expression profiles in our research were acquired from the Gene Expression Omnibus (GEO) database.

\section{Competing interests}

Not applicable

\section{Funding}

No external funds were used in this work.

\section{Authors' contributions}

Dr. Yang Hua participated in the data collection and data analysis. Dr. Jin-Yu Sun and Dr. Ziling Xin participated in the interpretation of data and drafted the manuscript. Dr. Wei Sun and Dr. Yanhui Sheng revised the initial manuscript. Dr. Xiang-Qing Kong participated in the manuscript submission.

\section{Acknowledgements}

None

\section{Conflicts of Interest}


The authors declare that they have no competing interests.

\section{References}

1. Maron, B.J., et al., Prevalence of hypertrophic cardiomyopathy in a general population of young adults. Echocardiographic analysis of 4111 subjects in the CARDIA Study. Coronary Artery Risk Development in (Young) Adults. Circulation, 1995. 92(4): p. 785-9.

2. Maron, B.J. and M.S. Maron, Hypertrophic cardiomyopathy. Lancet, 2013. 381(9862): p. 242-55.

3. Maron, B.J., Clinical Course and Management of Hypertrophic Cardiomyopathy. N Engl J Med, 2018. 379(7): p. 655-668.

4. Alcalai, R., J.G. Seidman, and C.E. Seidman, Genetic basis of hypertrophic cardiomyopathy: from bench to the clinics. J Cardiovasc Electrophysiol, 2008. 19(1): p. 104-10.

5. Seidman, C.E. and J.G. Seidman, Identifying sarcomere gene mutations in hypertrophic cardiomyopathy: a personal history. Circ Res, 2011. 108(6): p. 743-50.

6. Ackerman, M.J., et al., Prevalence and age-dependence of malignant mutations in the beta-myosin heavy chain and troponin T genes in hypertrophic cardiomyopathy: a comprehensive outpatient perspective. J Am Coll Cardiol, 2002. 39(12): p. 2042-8.

7. Niimura, $\mathrm{H}$., et al., Mutations in the gene for cardiac myosin-binding protein $C$ and late-onset familial hypertrophic cardiomyopathy. N Engl J Med, 1998. 338(18): p. 1248-57.

8. Van Driest, S.L., et al., Prevalence and severity of "benign" mutations in the beta-myosin heavy chain, cardiac troponin T, and alpha-tropomyosin genes in hypertrophic cardiomyopathy. Circulation, 2002. 106(24): p. 3085-90.

9. Richard, P., et al., Hypertrophic cardiomyopathy: distribution of disease genes, spectrum of mutations, and implications for a molecular diagnosis strategy. Circulation, 2003. 107(17): p. 222732.

10. Maron, B.J., M.S. Maron, and C. Semsarian, Genetics of hypertrophic cardiomyopathy after 20 years: clinical perspectives. J Am Coll Cardiol, 2012. 60(8): p. 705-15.

11. Landstrom, A.P. and M.J. Ackerman, Mutation type is not clinically useful in predicting prognosis in hypertrophic cardiomyopathy. Circulation, 2010. 122(23): p. 2441-9, discussion 2450.

12. Klues, H.G., et al., Diversity of structural mitral valve alterations in hypertrophic cardiomyopathy. Circulation, 1992. 85(5): p. 1651-60.

13. Maron, M.S., et al., Mitral valve abnormalities identified by cardiovascular magnetic resonance represent a primary phenotypic expression of hypertrophic cardiomyopathy. Circulation, 2011. 124(1): p. 40-7.

14. Aguiar Rosa, S., et al., Coronary microvascular dysfunction in hypertrophic cardiomyopathy: Pathophysiology, assessment, and clinical impact. Microcirculation, 2020: p. e12656.

15. Klues, H.G., A. Schiffers, and B.J. Maron, Phenotypic spectrum and patterns of left ventricular hypertrophy in hypertrophic cardiomyopathy: morphologic observations and significance as 
assessed by two-dimensional echocardiography in 600 patients. J Am Coll Cardiol, 1995. 26(7): p. 1699-708.

16. Horvath, S. and J. Dong, Geometric interpretation of gene coexpression network analysis. PLoS Comput Biol, 2008. 4(8): p. e1000117.

17. Langfelder, P. and S. Horvath, WGCNA: an R package for weighted correlation network analysis. BMC Bioinformatics, 2008. 9: p. 559.

18. Smyth, G.K., Linear models and empirical bayes methods for assessing differential expression in microarray experiments. Stat Appl Genet Mol Biol, 2004. 3: p. Article3.

19. Zhou, Y., et al., Metascape provides a biologist-oriented resource for the analysis of systems-level datasets. Nat Commun, 2019. 10(1): p. 1523.

20. Piñero, J., et al., DisGeNET: a comprehensive platform integrating information on human diseaseassociated genes and variants. Nucleic Acids Res, 2017. 45(D1): p. D833-d839.

21. Han, H., et al., TRRUST v2: an expanded reference database of human and mouse transcriptional regulatory interactions. Nucleic Acids Res, 2018. 46(D1): p. D380-d386.

22. Szklarczyk, D., et al., The STRING database in 2017: quality-controlled protein-protein association networks, made broadly accessible. Nucleic Acids Res, 2017. 45(D1): p. D362-d368.

23. Shannon, P., et al., Cytoscape: a software environment for integrated models of biomolecular interaction networks. Genome Res, 2003. 13(11): p. 2498-504.

24. Keren, A., P. Syrris, and W.J. McKenna, Hypertrophic cardiomyopathy: the genetic determinants of clinical disease expression. Nat Clin Pract Cardiovasc Med, 2008. 5(3): p. 158-68.

25. Marston, S.B., How do mutations in contractile proteins cause the primary familial cardiomyopathies? J Cardiovasc Transl Res, 2011. 4(3): p. 245-55.

26. Cahill, T.J., H. Ashrafian, and H. Watkins, Genetic cardiomyopathies causing heart failure. Circ Res, 2013. 113(6): p. 660-75.

27. Ingles, J., et al., Nonfamilial Hypertrophic Cardiomyopathy: Prevalence, Natural History, and Clinical Implications. Circ Cardiovasc Genet, 2017. 10(2).

28. Li, Y., et al., Expression of Vsig4 attenuates macrophage-mediated hepatic inflammation and fibrosis in high fat diet (HFD)-induced mice. Biochem Biophys Res Commun, 2019. 516(3): p. 858-865.

29. Li, J., et al., VSIG4 inhibits proinflammatory macrophage activation by reprogramming mitochondrial pyruvate metabolism. Nat Commun, 2017. 8(1): p. 1322.

30. Huang, X., et al., VSIG4 mediates transcriptional inhibition of NIrp3 and II-1 $\beta$ in macrophages. Sci Adv, 2019. 5(1): p. eaau7426.

31. Kitz, S., et al., Feline Hypertrophic Cardiomyopathy: The Consequence of Cardiomyocyte-Initiated and Macrophage-Driven Remodeling Processes? Vet Pathol, 2019. 56(4): p. 565-575.

32. Epelman, S., et al., Embryonic and adult-derived resident cardiac macrophages are maintained through distinct mechanisms at steady state and during inflammation. Immunity, 2014. 40(1): p. 91104. 
33. Ren, J., et al., Proinflammatory protein CARD9 is essential for infiltration of monocytic fibroblast precursors and cardiac fibrosis caused by Angiotensin II infusion. Am J Hypertens, 2011. 24(6): p. 701-7.

34. Wynn, T.A. and L. Barron, Macrophages: master regulators of inflammation and fibrosis. Semin Liver Dis, 2010. 30(3): p. 245-57.

35. Fallowfield, J.A., et al., Scar-associated macrophages are a major source of hepatic matrix metalloproteinase-13 and facilitate the resolution of murine hepatic fibrosis. J Immunol, 2007. 178(8): p. 5288-95.

36. Frangogiannis, N.G., Regulation of the inflammatory response in cardiac repair. Circ Res, 2012. 110(1): p. 159-73.

37. Law, S.K., et al., A new macrophage differentiation antigen which is a member of the scavenger receptor superfamily. Eur J Immunol, 1993. 23(9): p. 2320-5.

38. Daly, A., et al., Serum levels of soluble CD163 correlate with the inflammatory process in coeliac disease. Aliment Pharmacol Ther, 2006. 24(3): p. 553-9.

39. Boyle, J.J., et al., Coronary intraplaque hemorrhage evokes a novel atheroprotective macrophage phenotype. Am J Pathol, 2009. 174(3): p. 1097-108.

40. Etzerodt, A. and S.K. Moestrup, CD163 and inflammation: biological, diagnostic, and therapeutic aspects. Antioxid Redox Signal, 2013. 18(17): p. 2352-63.

41. Guo, L., et al., CD163+ macrophages promote angiogenesis and vascular permeability accompanied by inflammation in atherosclerosis. J Clin Invest, 2018. 128(3): p. 1106-1124.

42. Zhong, S.M., et al., [Clinical value of detecting serum soluble CD163 level in patients with atrial fibrillation]. Nan Fang Yi Ke Da Xue Xue Bao, 2016. 36(10): p. 1406-1409.

43. Watson, C.J., et al., Atrial Tissue Pro-Fibrotic M2 Macrophage Marker CD163+, Gene Expression of Procollagen and B-Type Natriuretic Peptide. J Am Heart Assoc, 2020. 9(11): p. e013416.

44. Ptaszynska-Kopczynska, K., et al., Alterations of soluble TWEAK and CD163 concentrations in patients with chronic heart failure. Cytokine, 2016. 80: p. 7-12.

45. Klimczak-Tomaniak, D., et al., Temporal patterns of macrophage- and neutrophil-related markers are associated with clinical outcome in heart failure patients. ESC Heart Fail, 2020. 7(3): p. 1190-1200.

46. Graversen, J.H., et al., Targeting the hemoglobin scavenger receptor CD163 in macrophages highly increases the anti-inflammatory potency of dexamethasone. Mol Ther, 2012. 20(8): p. 1550-8.

47. Gleissner, C.A., et al., CXCL4 downregulates the atheroprotective hemoglobin receptor CD163 in human macrophages. Circ Res, 2010. 106(1): p. 203-11.

48. Allen, R.D., et al., Surgical pathology of subaortic septal myectomy not associated with hypertrophic cardiomyopathy: a study of 98 cases (1996-2000). Cardiovasc Pathol, 2003. 12(4): p. 207-15.

49. Baandrup, U. and E.G. Olsen, Critical analysis of endomyocardial biopsies from patients suspected of having cardiomyopathy. I: Morphological and morphometric aspects. Br Heart J, 1981. 45(5): p. 47586. 
50. Fang, L., et al., Systemic inflammation is associated with myocardial fibrosis, diastolic dysfunction, and cardiac hypertrophy in patients with hypertrophic cardiomyopathy. Am J Transl Res, 2017. 9(11): p. 5063-5073.

51. Brandsma, A.M., et al., Clarifying the Confusion between Cytokine and Fc Receptor "Common Gamma Chain". Immunity, 2016. 45(2): p. 225-6.

52. Haudek, S.B., et al., Fc receptor engagement mediates differentiation of cardiac fibroblast precursor cells. Proc Natl Acad Sci U S A, 2008. 105(29): p. 10179-84.

53. van Beek, L., et al., FcRY-chain deficiency reduces the development of diet-induced obesity. Obesity (Silver Spring), 2015. 23(12): p. 2435-44.

54. Shimizu, I. and T. Minamino, Physiological and pathological cardiac hypertrophy. J Mol Cell Cardiol, 2016. 97: p. 245-62.

55. Zheng, L., et al., Role and mechanism of cardiac insulin resistance in occurrence of heart failure caused by myocardial hypertrophy. Aging (Albany NY), 2019. 11(16): p. 6584-6590.

56. Pak, Y., et al., Transport of LAPTM5 to lysosomes requires association with the ubiquitin ligase Nedd4, but not LAPTM5 ubiquitination. J Cell Biol, 2006. 175(4): p. 631-45.

57. Ouchida, R., et al., A lysosomal protein negatively regulates surface $T$ cell antigen receptor expression by promoting CD3zeta-chain degradation. Immunity, 2008. 29(1): p. 33-43.

58. Ouchida, R., T. Kurosaki, and J.Y. Wang, A role for lysosomal-associated protein transmembrane 5 in the negative regulation of surface $B$ cell receptor levels and B cell activation. J Immunol, 2010. 185(1): p. 294-301.

59. Kawano, Y., et al., A novel mechanism for the autonomous termination of pre-B cell receptor expression via induction of lysosome-associated protein transmembrane 5. Mol Cell Biol, 2012. 32(21): p. 4462-71.

60. Glowacka, W.K., et al., LAPTM5 protein is a positive regulator of proinflammatory signaling pathways in macrophages. J Biol Chem, 2012. 287(33): p. 27691-702.

61. Inoue, J., et al., Lysosomal-associated protein multispanning transmembrane 5 gene (LAPTM5) is associated with spontaneous regression of neuroblastomas. PLoS One, 2009. 4(9): p. e7099.

62. Nuylan, M., et al., Down-regulation of LAPTM5 in human cancer cells. Oncotarget, 2016. 7(19): p. 28320-8.

\section{Figures}




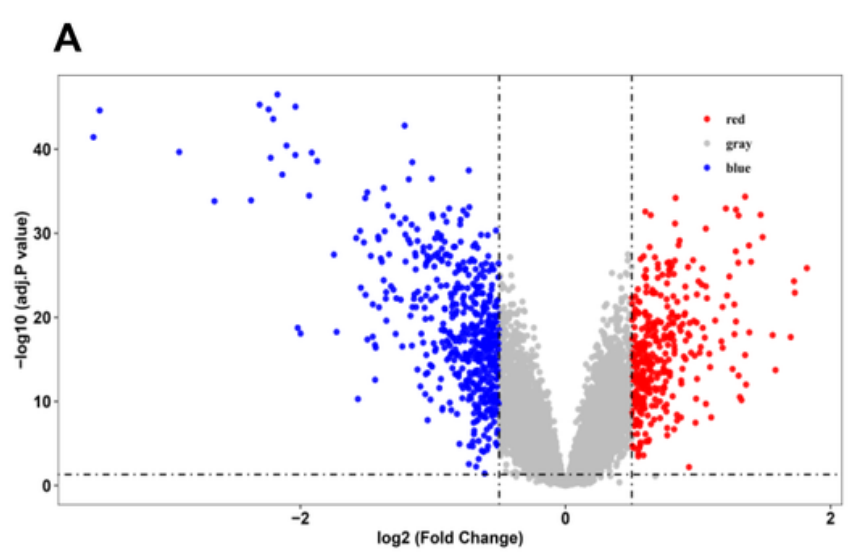

B
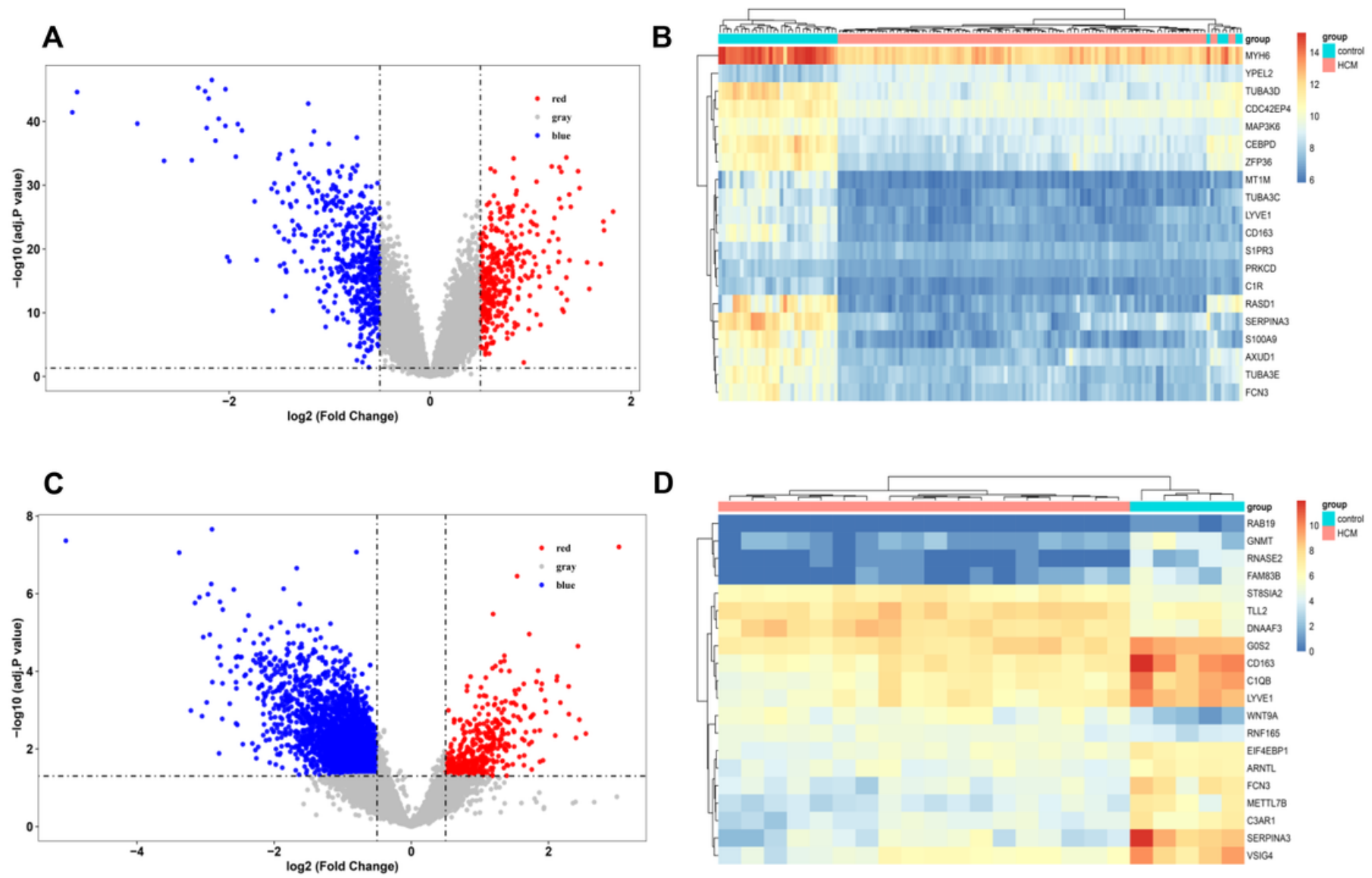

D

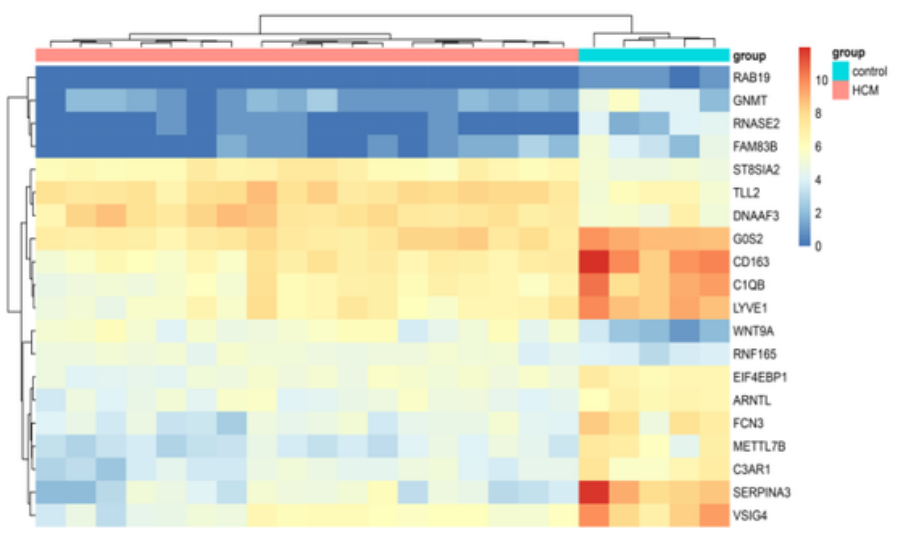

Figure 1

Volcano plots and heatmaps of the differential gene expression data between hypertrophic cardiomyopathy and normal samples from (A-B) GSE36961, (C-D) GSE160997. In the volcano plots, the red points show up-regulated genes ( $\log 2 F C \geq 0.5$ and adjusted $P$ value $<0.05$ ), and the blue points represent down-rftableegulated genes ( $\log 2 F C \geq 0.5$ and adjusted $P$ value $<0.05$ ). FC: fold-change. 

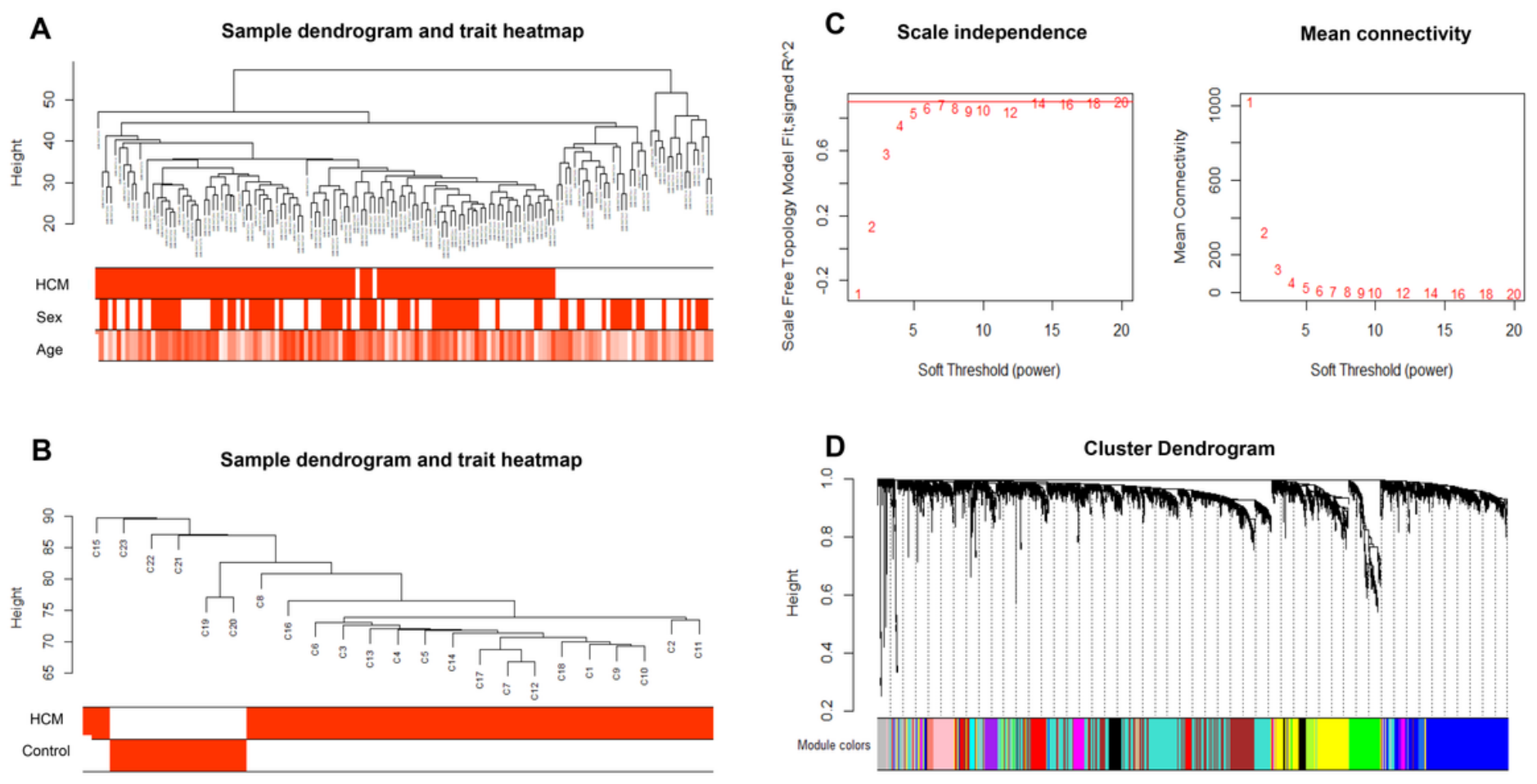

\section{Figure 2}

Sample clustering and network construction of the weighted co-expressed genes. (A) Clustering dendrogram of samples from GSE36961. (B) Clustering dendrogram of samples from GSE160997. The color intensity was proportional to disease status (hypertrophic cardiomyopathy or normal tissue), age, and sex. (C) Analysis of the scale-free fit index and the mean connectivity for various soft-thresholding powers. The soft-thresholding power of 7 was selected based on the scale free topology criterion. (D) Dendrogram clustered based on a dissimilarity measure (1-TOM). Each color below represents one coexpression module, and every branch stands for one gene. 


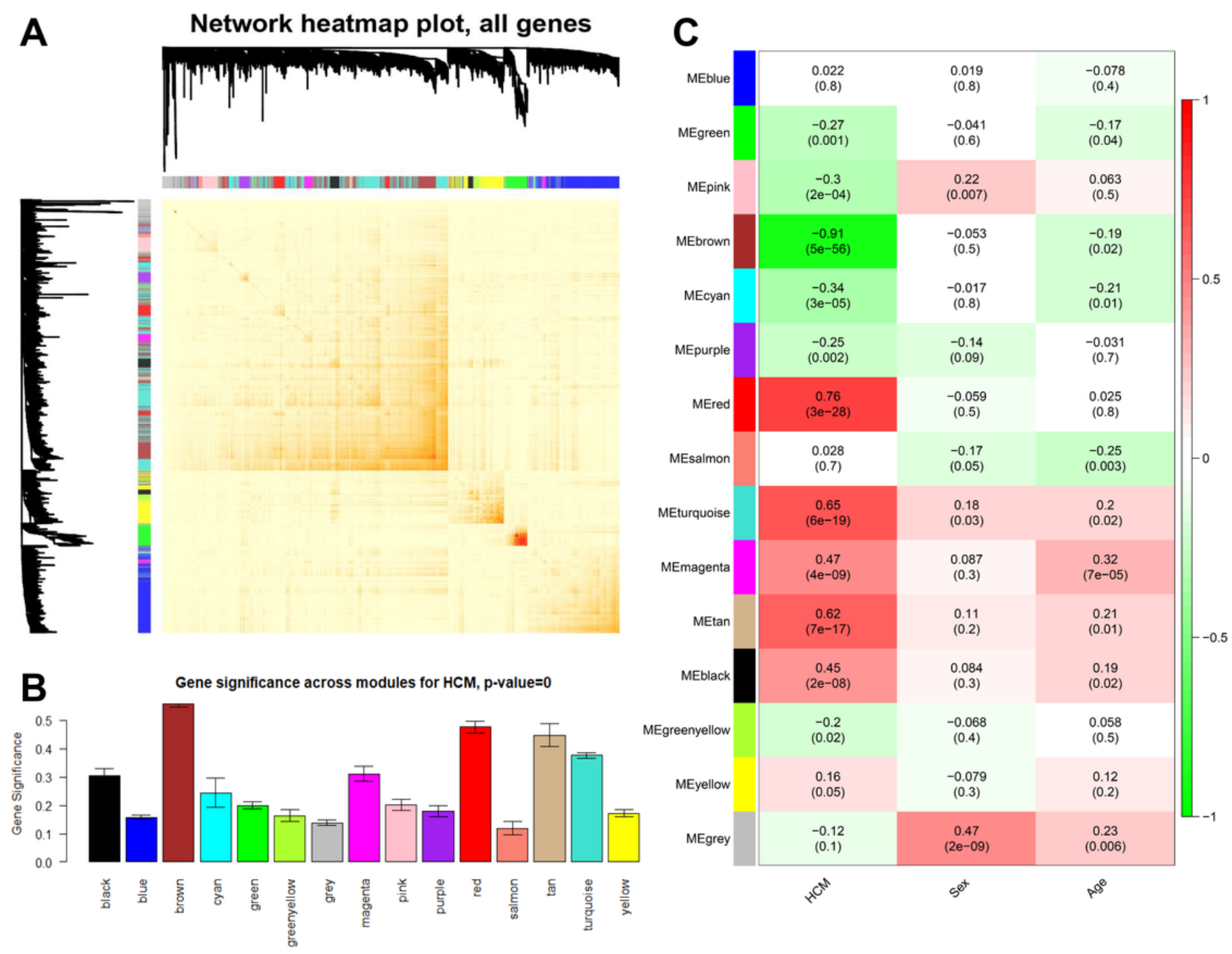

\section{Figure 3}

Association analysis of modules. (A) Different colors represent different modules, and the density of yellow in the middle of the figure represents the connectivity of different modules. There was no significant dissimilarity of association among different modules, showing high-degree independence among these modules. (B) Distribution of gene significance in the modules associated with HCM. The red and brown modules were the most significantly correlated with disease status. (C) Heatmap of the correlation between module eigengenes and the disease status, age and sex of HCM. 
A

Module membership vs gene significance cor $=0.78, p=2.9 e-46$

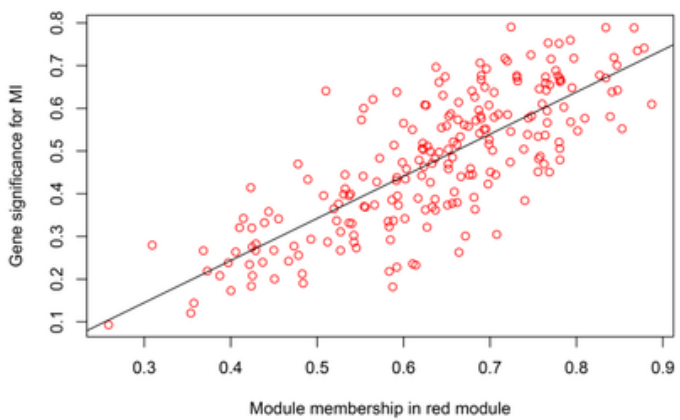

C

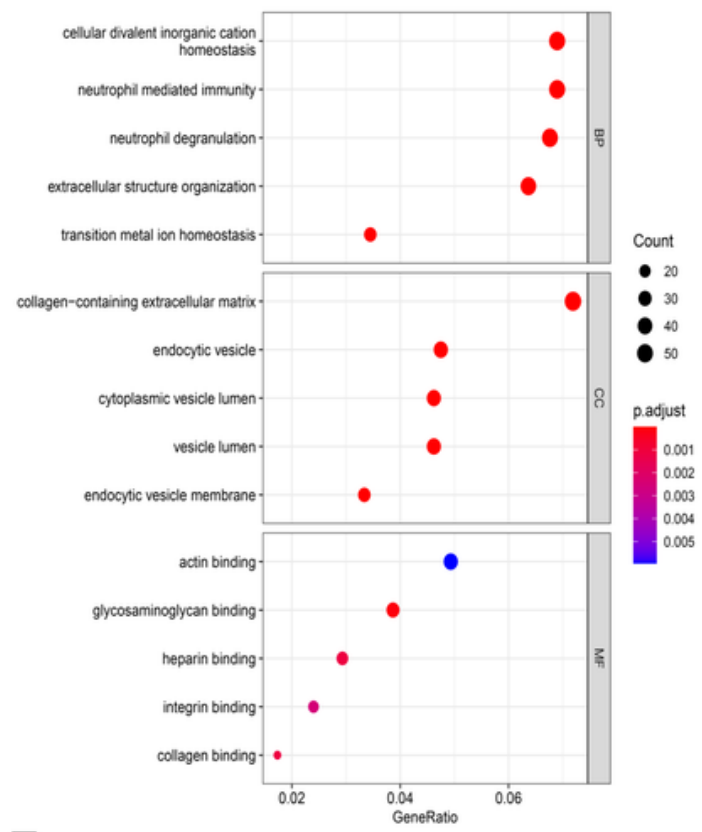

E

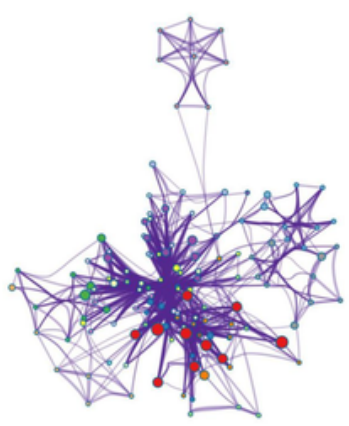

B

Module membership vs gene significance cor $=0.95, p<1 e-200$

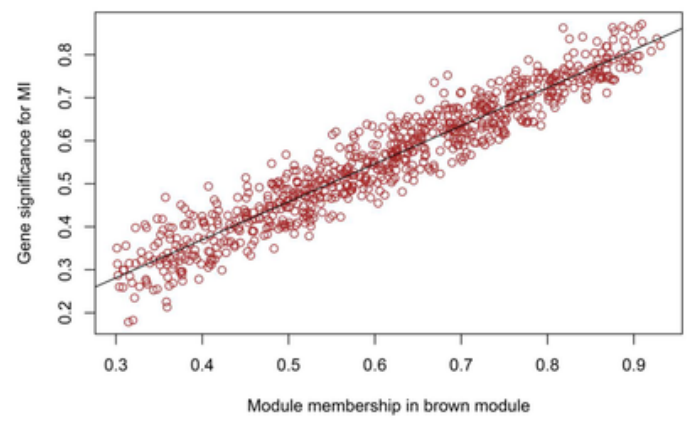

D

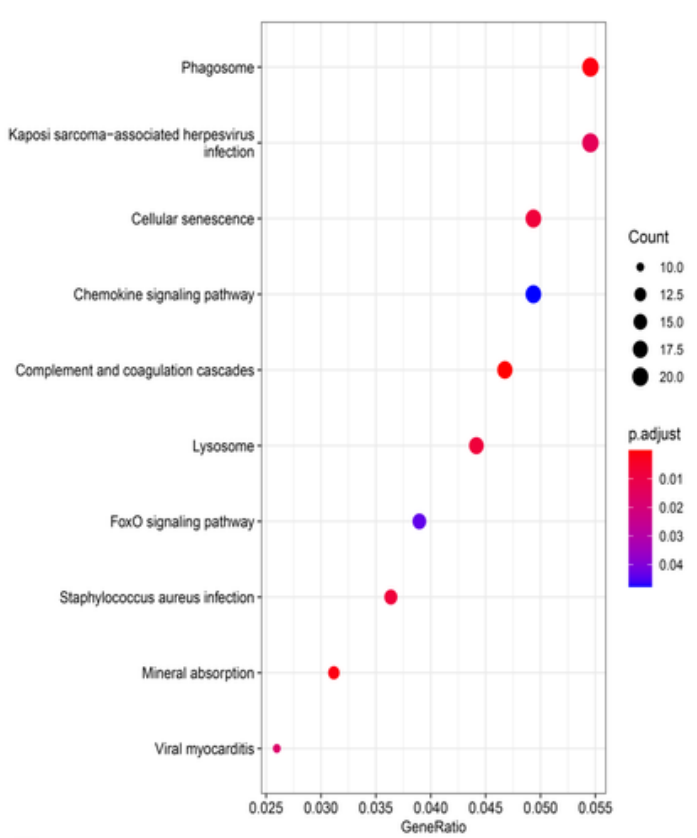

$\mathbf{F}$

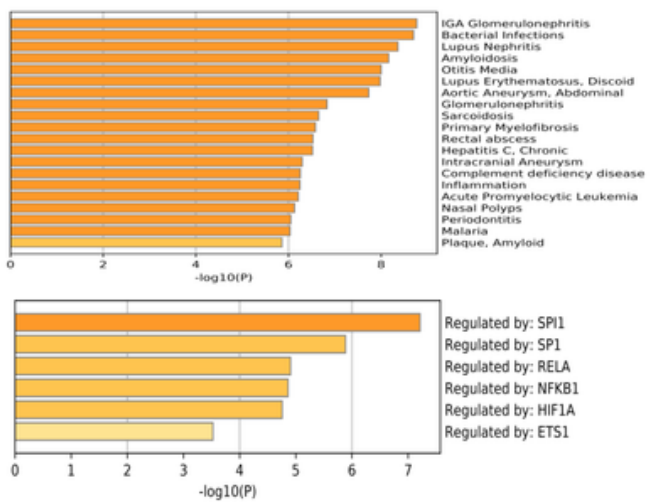

\section{Figure 4}

Scatter plot of module eigengenes in the (A) red module and (B) brown module. (C-D) Gene ontology and KEGG pathway enrichment analysis in red and brown modules. The significance of enrichment gradually increases from blue to red, and the size of the dots indicates the number of genes contained in the corresponding pathway. (E) The association between genes and pathways visualized by Metascape. (F) The enrichment analysis of module genes performed in DisGeNET and TRRUST database. 
A

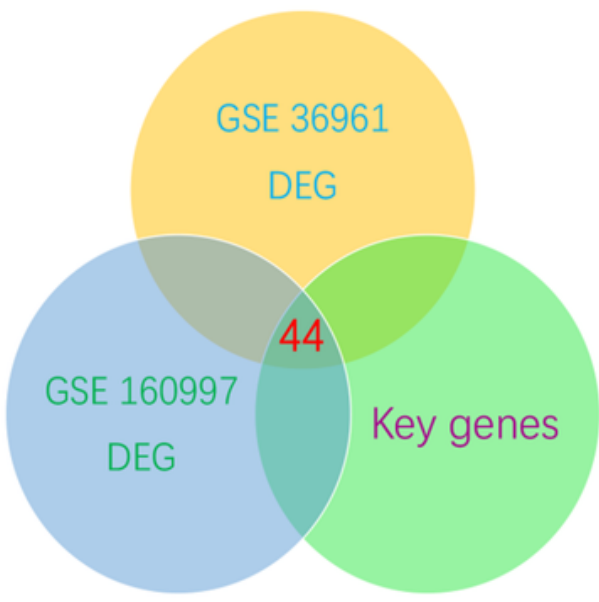

C

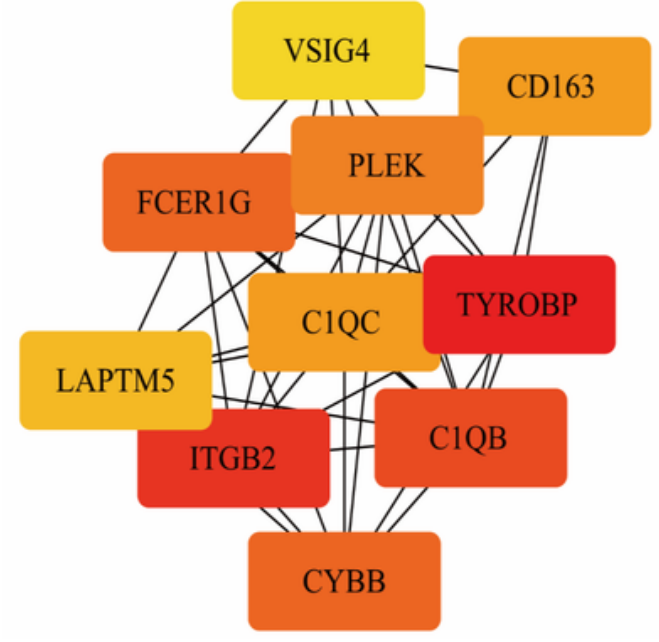

B

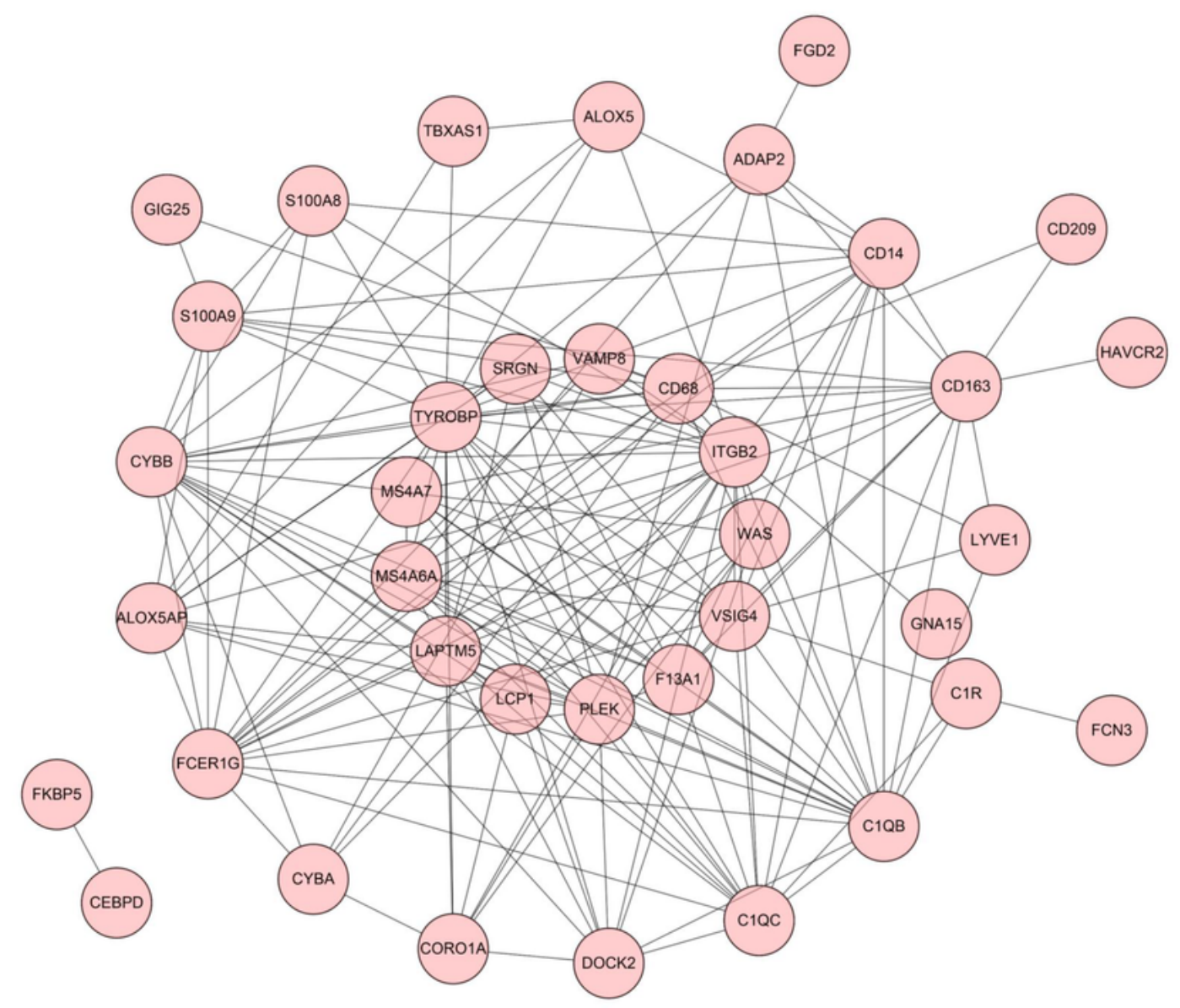

Figure 5

(A) The venn diagram of key genes from the red and brown modules and DEGs from GSE36961, and GSE160997. (B) The protein-protein interaction network of the overlapped genes. (C) 5 hub genes identified by 'cytoHubba' according to calculation of degree connectivity. 